\title{
L-ARGININE POLYMERS INHIBIT THE DEVELOPMENT OF VEIN GRAFT NEOINTIMAL HYPERPLASIA
}

Murray H. Kown, MD

Atsushi Yamaguchi, MD

Christina L. Jahncke ${ }^{\mathrm{a}}$

Douglas Miniati, MD

Seiichiro Murata, MD

Jurg Grunenfelder, MD

Mark L. Koransky, MD

Jonathan B. Rothbard, $\mathrm{PhD}^{\mathrm{b}}$

Robert C. Robbins, $\mathrm{MD}^{\mathrm{a}}$
Objective: We sought to determine whether L-arginine polymer treatment of vein grafts enhances vascular production of nitric oxide and inhibits the development of neointimal hyperplasia.

Methods: External jugular veins of New Zealand White rabbits $(n=42)$ were harvested; treated intraluminally for 15 minutes with phosphate-buffered saline solution or L-arginine polymer 5,7 , or 9 at either 10 or $100 \mu \mathrm{mol} / \mathrm{L}$; and then grafted into the contralateral carotid artery. Rabbits were killed after 28 days, and 5- $\mu \mathrm{m}$ sections of vessels were stained with hematoxylin and scored for intima/media ratio by using computerized morphometric analysis. Separate veins were treated in a similar fashion with biotinylated polymers and phosphate-buffered saline solution to assess for translocation efficiencies. Finally, vein segments pretreated with either phosphatebuffered saline solution or L-arginine polymers were cultured in Dulbecco's modified Eagle's medium containing lipopolysaccharide $(100 \mu \mathrm{g} / \mathrm{mL})$ and interferon $\gamma(200 \mathrm{U} / \mathrm{mL})$ for 48 hours before measuring nitric oxide levels by means of the Griess reaction.

Results: Biotinylated L-arginine polymers demonstrated a dose- and lengthdependent uptake into intimal and medial cells of treated vessels. Nitric oxide levels were significantly higher in vein segments treated with 100 $\mu \mathrm{mol} / \mathrm{L}$ of L-arginine polymer 9 compared with control segments. Finally, the intima/media ratio also reflected both length- and concentration-dependent inhibition of neointimal hyperplasia.

Intima/media ratio

\begin{tabular}{lccll}
\hline & $P B S$ & \multicolumn{1}{c}{$R 5$} & \multicolumn{1}{c}{$R 7$} & \multicolumn{1}{c}{$R 9$} \\
\hline $10 \mu \mathrm{mol} / \mathrm{L}$ & $0.909 \pm 0.072$ & $0.920 \pm 0.073$ & $0.861 \pm 0.138$ & $0.710 \pm 0.122$ \\
$100 \mu \mathrm{mol} / \mathrm{L}$ & & $0.924 \pm 0.061$ & $0.581 \pm 0.089^{*}$ & $0.529 \pm 0.093^{*}$
\end{tabular}

$P B S$, Phosphate-buffered saline solution; $R$, L-arginine polymer.

$* P<.001$ versus phosphate-buffered saline solution and L-arginine polymer 5 controls (Bonferronicorrected value).

Conclusions: Arginine polymers of sufficient length and concentration were effective in increasing nitric oxide levels and reducing neointimal hyperplasia in this vein graft model. (J Thorac Cardiovasc Surg 2001;121:971-80)
From the Department of Cardiothoracic Surgery, ${ }^{\text {a }}$ Stanford University School of Medicine, Stanford, Calif; and Cellgate, ${ }^{b}$ Sunnyvale, Calif.

Supported by the Roche Laboratories Surgical Scientist Scholarship from the American Society of Transplant Surgeons and the Ralph and Marian Falk Foundation for Cardiovascular Research.

Read at the Twenty-sixth Annual Meeting of The Western Thoracic Surgical Association, The Big Island, Hawaii, June 21-24, 2000.

Copyright (c) 2001 by The American Association for Thoracic Surgery

$0022-5223 / 2001 \$ 35.00+0 \quad \mathbf{1 2 / 6 / 1 1 2 5 3 2}$

doi: $10.1067 / \mathrm{mtc} .2001 .112532$
D espite concerted efforts to increase the use of arterial grafts in recent years, saphenous veins remain the most widely used bypass vessels in coronary artery revascularization procedures. ${ }^{1}$ Unfortunately, venous conduits develop accelerated atherosclerosis as a result of neointimal hyperplasia. This is partially responsible for the progressive occlusion that limits 15-year patencies to approximately $50 \% .^{2}$

Vein graft injury initially results from increased wall stresses incurred by arterial pressures, transient is- 

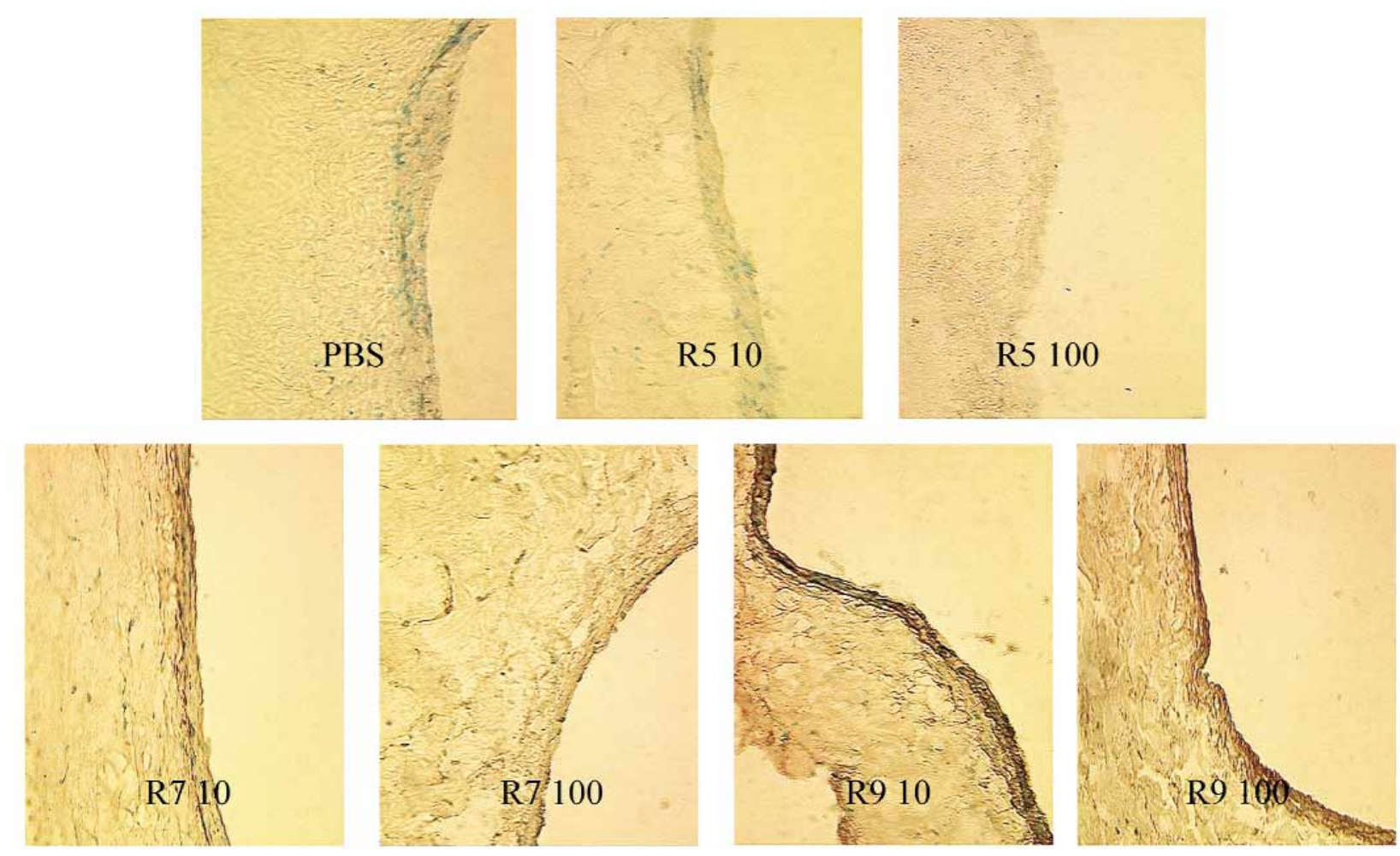

Fig 1. Representative figures of biotinylated L-arginine polymer and PBS-treated rabbit external jugular veins. Vessel lumina are on the right side of each photomicrograph. Nuclei are visible as light green areas, whereas dark brown regions signify areas of $\mathrm{L}$-arginine polymer uptake. There is minimal biotin staining apparent in either PBS or R5 groups in contrast to the darkened nuclei and cytoplasm of R7 and R9 categories. (Original magnification, 400×.)

chemia followed by reperfusion after grafting, and traumatic endothelial cell loss caused by manipulation. These lead to the liberation of growth factors and cytokines that influence the migration and proliferation of vascular smooth muscle cells (VSMCs), as well as the deposition of extracellular matrix into the intimal compartment of the affected vessels. ${ }^{3}$

Nitric oxide (NO) is an important vasoactive molecule that limits neointimal hyperplasia by inhibiting VSMC proliferation and inducing cellular death by apoptosis. ${ }^{4,5}$ It is a short-lived molecule that is formed by the activity of nitric oxide synthase (NOS) on its substrate, L-arginine. Although constitutive NOS is present in endothelial and neuronal cells, a third isoform, inducible NOS (iNOS), is expressed as a compensatory mechanism in vascular injury. ${ }^{6}$ The synthesis of NO during these periods of elevated iNOS appears to be substrate limited, ${ }^{7}$ and indeed, numerous investigators have used L-arginine administration to increase NO production during conditions of vascular stress. ${ }^{8-10}$

It has recently been noted that short polymers of Larginine of 6 amino acids or greater in length are capable of translocating efficiently across cytoplasmic membranes of vascular cells. ${ }^{11}$ Once located within the cell, these polymers can provide the L-arginine substrate for iNOS production of NO. The objective of this study was thus to determine whether L-arginine polymer treatment of vein grafts can enhance the production of vascular NO and inhibit the ultimate development of neointimal hyperplasia.

\section{Methods}

Peptide synthesis. Arginine polymers were synthesized on an Applied Biosystems 433 peptide synthesizer by using previously described solid-phase techniques and commercially available Fmoc amino acids, resins, and reagents (Applied Biosystems, Foster City, Calif, and Bachem, Torrance, Calif). ${ }^{12}$ The following polymer sequences were used: L-arginine polymer (R) 5, $\mathrm{NH}_{2}-\mathrm{RRRRR}-\mathrm{CONH}_{2} ; \mathrm{R} 7, \mathrm{NH}_{2}$-RRRRRRR$\mathrm{CONH}_{2} ; \mathrm{R} 9, \mathrm{NH}_{2}-\mathrm{RRRRRRRRR-CONH}{ }_{2}$; and phosphatebuffered saline (PBS) solution. Biotinylated polymers of each length were used in studies of translocation efficiency.

Animals. Male New Zealand White rabbits weighing between 2.5 and $3.5 \mathrm{~kg}$ were purchased from Krelek Farms (Stockton, Calif) and housed at the animal care facilities at the Department of Cardiothoracic Surgery, Stanford University Medical Center (Stanford, Calif). They were kept 


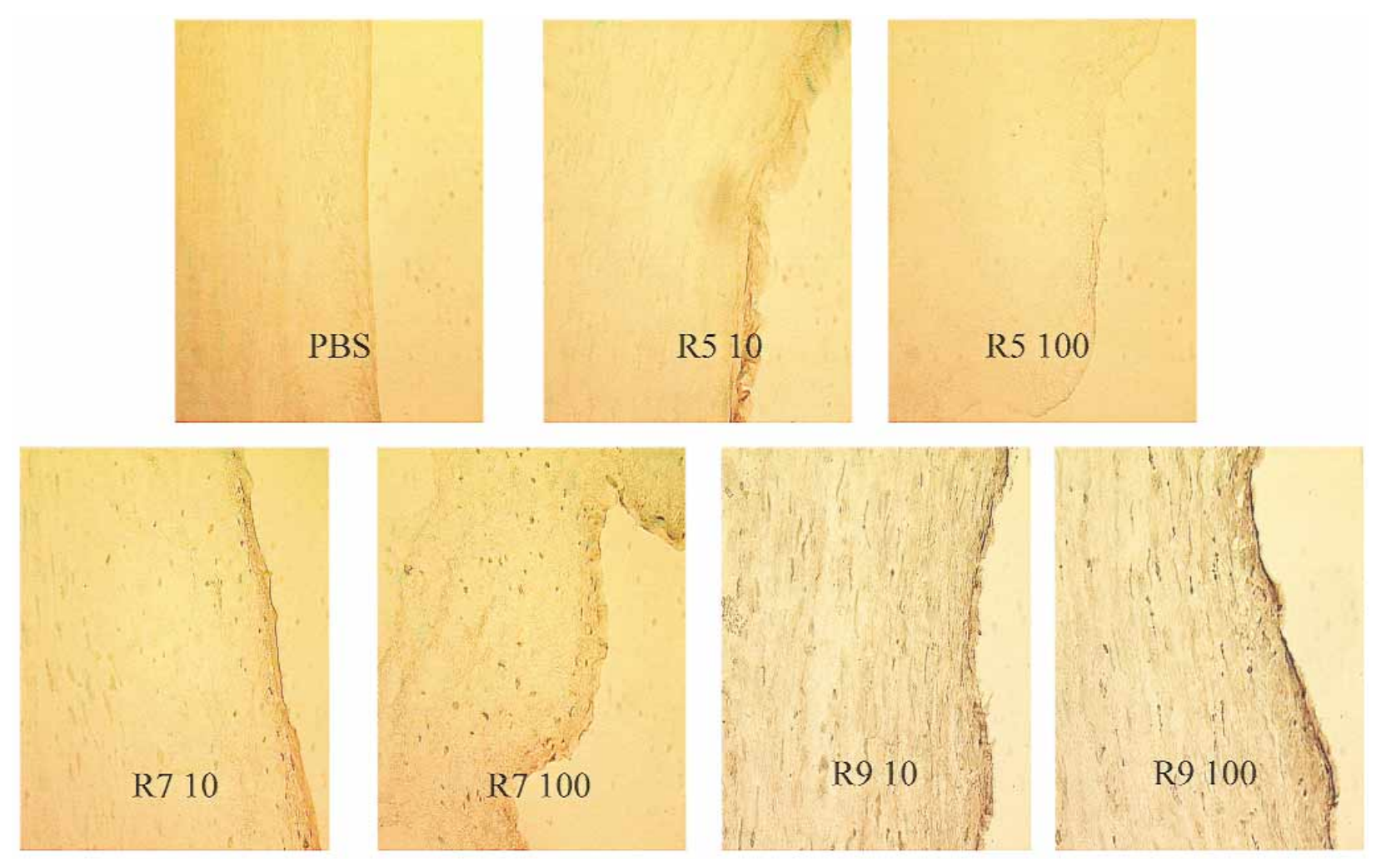

Fig 2. Representative figures of biotinylated L-arginine polymer and PBS-treated human saphenous veins. As is the case with rabbit veins, there appears to be little or no uptake of the polymers in either the PBS or R5 groups, whereas considerable expression of biotin is noted in the intima and media of vessels in both the R7 and R9 groups. (Original magnification, 400×.)

in rooms under normal temperature, humidity, and timed lighting conditions and were provided standard food and water ad libitum. Animals were treated in a humane manner in compliance with the "Principles of Laboratory Animal Care" formulated by the National Society for Medical Research and the "Guide for the Care and Use of Laboratory Animals" prepared by the Institute of Laboratory Animal Resources, National Research Council, and published by the National Academy Press, revised 1996.

Surgical procedure. After anesthesia with intramuscular injection of $50 \mathrm{mg} / \mathrm{kg}$ ketamine and $5 \mathrm{mg} / \mathrm{kg}$ xylazine and mask inhalation of $2.0 \%$ isoflurane, the external jugular vein was isolated through a midline longitudinal neck incision. The vein segment was flushed of blood with normal saline solution and then instilled with either PBS, R5, R7, or R9 in solutions with concentrations of 10 or $100 \mu \mathrm{mol} / \mathrm{L}$ for 15 minutes $(n=6$ per group). The segments were then reversed and anastomosed as an interposition graft in an end-to-side fashion to the contralateral carotid artery. The carotid artery segment between the proximal and distal anastomoses was then ligated and transected. Animals were killed after 28 days, and the vein grafts were perfusion fixed with $10 \%$ buffered formalin solution.

Translocating efficiency. To demonstrate the efficiency of arginine polymer penetration of vascular cells in vivo, the external jugular veins of New Zealand White rabbits were iso- lated as above and incubated for 15 minutes with either PBS or biotinylated R5, R7, or R9 in solutions of 10 or $100 \mu \mathrm{mol} / \mathrm{L}$ concentrations ( $n=1$ per group). These were then flushed with PBS solution before freezing in OCT compound and cutting into $5-\mu \mathrm{m}$ sections. Frozen sections were fixed in acetone for 10 minutes at $-20^{\circ} \mathrm{C}$, washed in PBS solution for 3 separate 5minute intervals, and incubated for 30 minutes with peroxidase suppressor (Pierce Chemical Co, Rockford, Ill) to block endogenous activity. Slides were then washed in PBS solution as above and incubated with $5 \mu \mathrm{g} / \mathrm{mL}$ horseradish peroxidaseconjugated streptavidin (Pierce Chemical Co) for 30 minutes. They were then washed and incubated for 60 seconds with the horseradish peroxidase substrate 3,3'-diaminobenzidine (Sigma Chemical Company, St Louis, Mo). Reactions were terminated by rinsing slides in distilled water before methyl green counterstaining and dehydration in ethanol.

Because of the relatively thin media in native rabbit veins, the same experiments were conducted in unused human saphenous vein segments obtained from patients undergoing coronary artery bypass grafting. The thicker vessel walls of the human vein segments allowed better appreciation of medial uptake. One vessel was treated per group, and 3 random highpower fields per cross section were analyzed for translocation of polymers. A quantitative measure of efficiency was calculated by dividing biotin-staining nuclei by total nuclei for both 


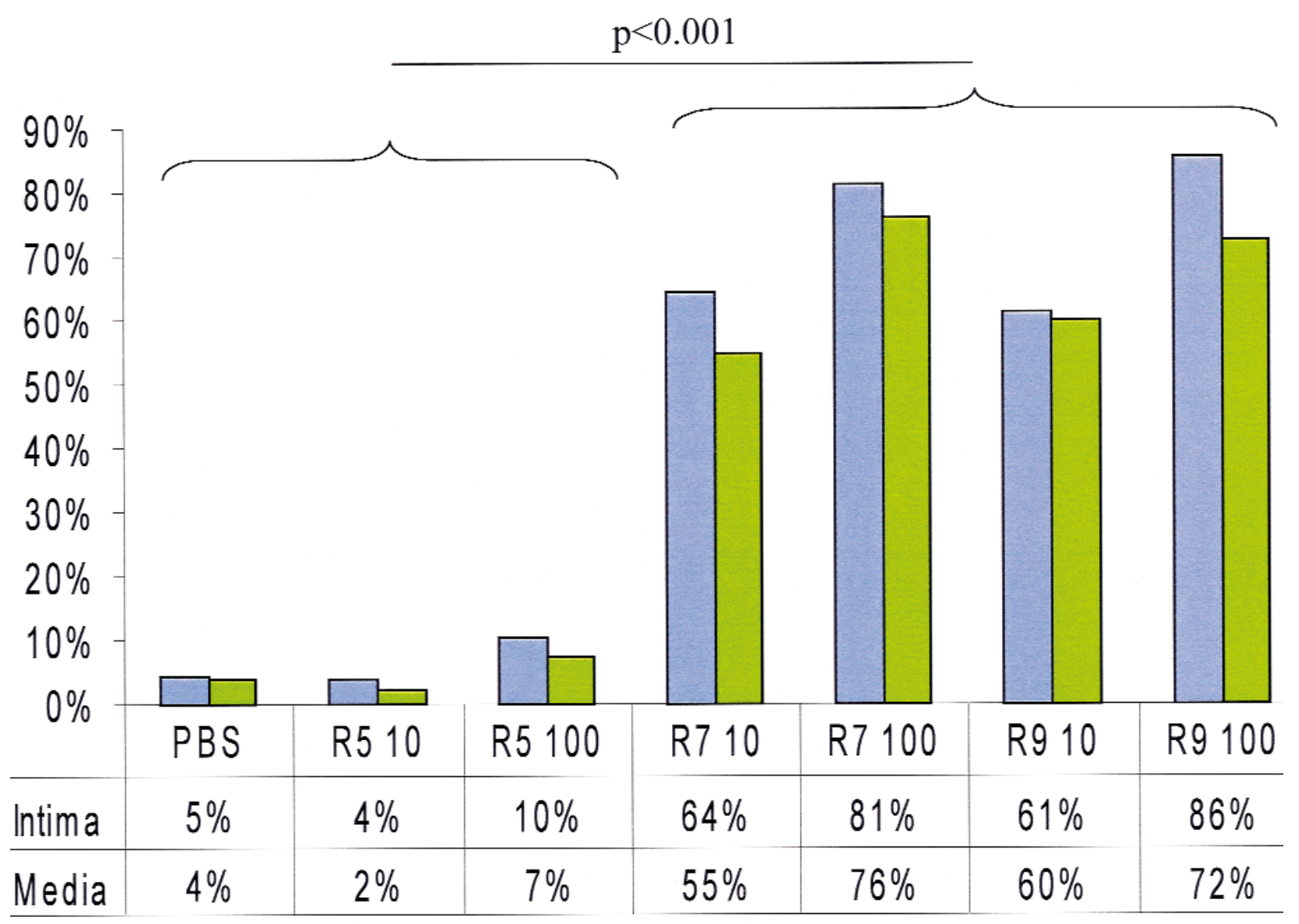

Fig 3. Quantitative measure of translocation efficiencies on the basis of the number of biotin-staining nuclei (brown) divided by the number of total nuclei (brown plus green) in human saphenous vein samples. Each bar represents the average of 3 random high-power field readings of a cross-section of a single vessel. One hundred micromolar concentration appears to translocate with the highest efficiencies. Average translocation of the PBS and R5 group was significantly lower than that of R7 and R9 $(P<.001$, Student $t$ test).

intimal and medial regions of these vessels, and an average of the 3 readings was taken. Statistical comparisons were made between a group comprised of PBS and R5 categories $(n=3)$, where little or no translocation was seen, and a group consisting of R7 and R9 categories $(n=4)$, where high translocation was present. The Medical Human Subjects Panel at Stanford University approved the use of human tissues in this study.

NO assay. Efficacy of L-arginine polymer therapy was assessed by means of in vitro assays for NO production. Separate rabbit external jugular veins were sectioned and treated with PBS solution or $100 \mu \mathrm{mol} / \mathrm{L}$ solutions of R5, R7, or $\mathrm{R} 9$ for 15 minutes ( $\mathrm{n}=6$ per group). The vein sections were then incubated in Dulbecco's modified Eagle's medium (Gibco BRL, Gaithersburg, Md) containing both lipopolysaccharide (LPS; $100 \mu \mathrm{g} / \mathrm{mL}$ ) and interferon (IFN) $\gamma(200 \mathrm{U} / \mathrm{mL}$; Sigma Chemical Co) to upregulate iNOS production. After 48 hours' incubation at $37^{\circ} \mathrm{C}$, nitrite $\left(\mathrm{NO}_{2}^{-}\right)$accumulation in the cell culture medium was directly quantified by the Griess reaction through a commercially available colorimetric assay
(Cayman Chemical, Ann Arbor, Mich). Values were measured as micromolar concentrations and standardized to the weight of venous tissues in milligrams.

Vessel morphometry. Finally, the biologic effect of Larginine polymer therapy was assessed by measuring the degree of neointimal hyperplasia in the 28-day treated and control vein grafts. The vein segments were placed in paraffin blocks, and the middle portion of each specimen was cut into 5- $\mu \mathrm{m}$ sections for staining with hematoxylin dye. A computerized morphometric analysis program (C-Imaging Systems, Cranberry Township, NJ) was used to measure intimal and medial areas of one section per graft in a blinded fashion. Neointimal hyperplasia is expressed as a ratio of these two measures (intima/media ratio $[\mathrm{I} / \mathrm{M}]$ ).

Statistical analysis. Values are expressed as means \pm 1 SD. All comparisons were made by 1-way analysis of variance (ANOVA; SYSTAT Inc, Chicago, Ill). Unpaired Student $t$ tests were used to identify statistical significance between groups, with the Bonferroni correction for multiple comparisons. 


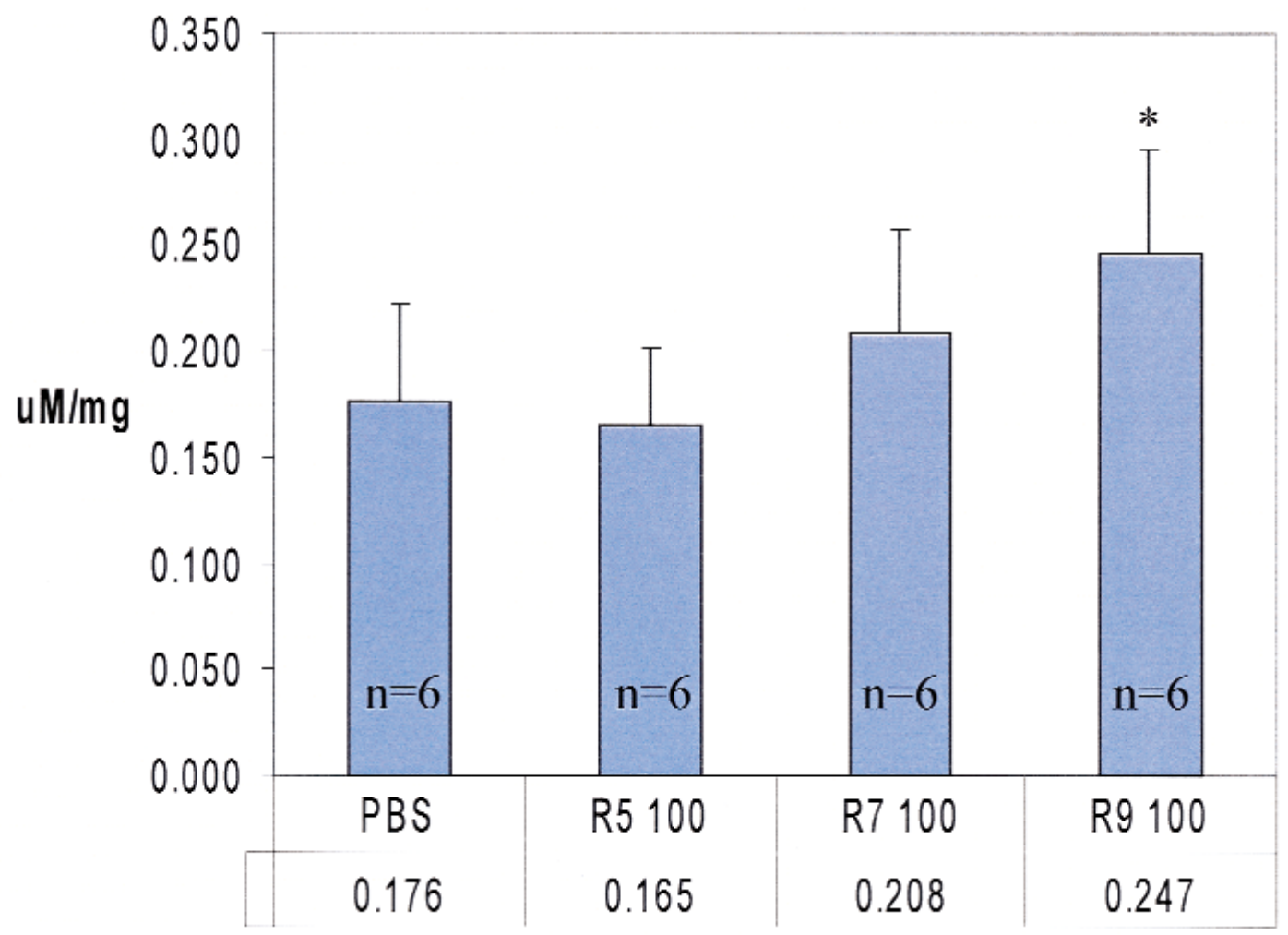

Fig 4. NO production as measured by nitrite $\left(\mathrm{NO}_{2}^{-}\right)$levels. Pretreated venous segments are incubated in culture medium containing LPS $(100 \mu \mathrm{g} / \mathrm{mL})$ and IFN- $\gamma(200 \mu \mathrm{g} / \mathrm{mL})$ for 48 hours. Nitrite levels are measured directly from culture medium by means of Griess reaction and standardized to weights of venous segments. There is a length-dependent increase in nitrite levels with $\mathrm{L}$-arginine polymer treatment. $* P=.033$ versus $\mathrm{R} 5100 \mu \mathrm{mol} / \mathrm{L}$ and $P=.085$ versus PBS (Bonferonni-corrected value).

\section{Results}

Arginine polymer uptake efficiencies. Microscopic examination of rabbit jugular veins treated with biotinylated L-arginine polymers and PBS solution did reveal length-dependent uptake. Although R7- and R9-treated specimens exhibited evidence of vascular cell penetration to the level of the adventitia, neither the PBS nor R5 groups showed appreciable staining (Fig 1).

Translocation efficiencies were estimated in human saphenous vein segments because of the thicker medial layer allowing a more detailed analysis of uptake (Figs 2 and 3). R7 and R9 concentrations of 100 $\mu \mathrm{mol} / \mathrm{L}$ resulted in the highest efficiencies at $81 \%$ and $86 \%$, respectively. The intimal regions had greater uptake than their medial counterparts because of their direct exposure to treatment solutions. Neither PBS nor $100 \mu \mathrm{mol} / \mathrm{L}$ R5 groups had appreciable translocations. Average intimal translocation efficiencies were $6 \% \pm$ $3 \%$ for the PBS and R5 groups versus $73 \% \pm 12 \%$ for the R7 and R9 categories $(P<.001$, Student $t$ test).

Measure of NO production. The effects of L-arginine polymers on venous production of $\mathrm{NO}$ were measured in vitro. Cytokine (IFN- $\gamma$ ) and endotoxin (LPS) stimulation were used to upregulate iNOS levels ${ }^{13}$ in vein segments pretreated with either PBS solution or L-arginine polymers. Direct measurement of culture medium for the NO byproduct, nitrite $\left(\mathrm{NO}_{2}^{-}\right)$, revealed a length-dependent effect of the polymers at 48 hours with the $\mathrm{R} 9$ group having the highest levels ( $P=.024$, ANOVA; Fig 4$)$. There was also an incremental, although statistically insignificant, increase in NO in the R7-treated group when compared with either PBS or R5 solution.

Measure of neointimal hypertrophy. All animals survived until the time of harvest, at which point $88 \%$ of the grafts were noted to be patent by means of direct examination. There was no preponderance of early occlusion in any of the study groups. Although each vessel did have a degree of concentric neointimal hyperplasia (Fig 5, $A$ and $B$ ), I/M ratios, as measured by means of computerized morphometric analysis, did demonstrate a protective effect of L-arginine polymers of sufficient length and concentration. One-way ANOVA yielded a highly significant difference among groups $(P<.001)$. The I/M ratio in the PBS $(0.91)$ and 


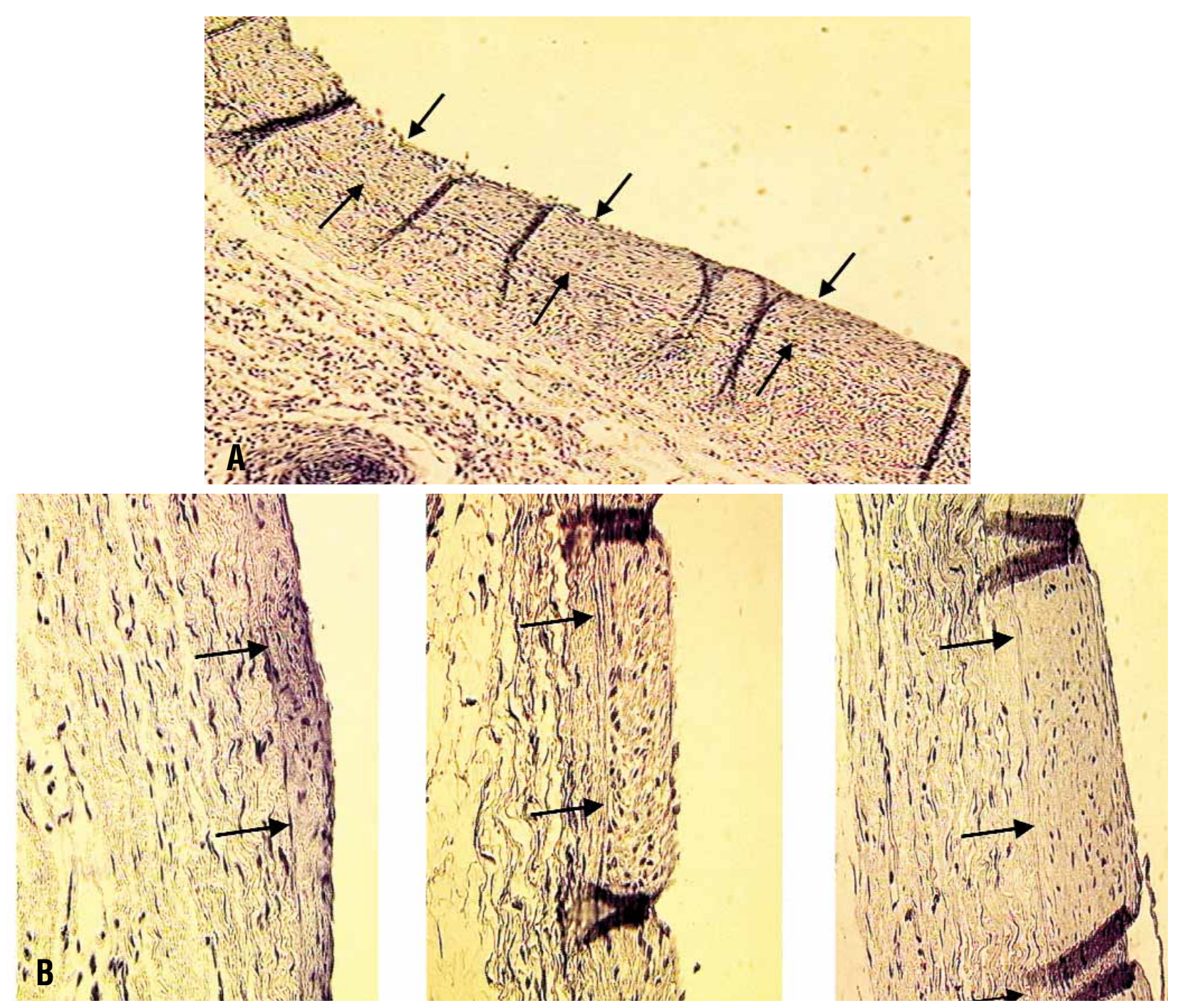

Fig 5. A, Low-magnification view (100x) of the concentric nature of neointimal hyperplasia visible at 28 days in vein grafts stained with hematoxylin. Arrows delineate the neointimal layer. B, Higher magnification view $(400 \times)$ of low, medium, and high degrees of neointimal hyperplasia. Arrows depict the border between medial and neointimal layers.

both R5 groups $(10 \mu \mathrm{mol} / \mathrm{L} \mathrm{R}$ 5, 0.92; $100 \mu \mathrm{mol} / \mathrm{L}$ R5, 0.92 ) were relatively consistent. A minimal reduction in neointimal hyperplasia was seen at the lowest concentration of R7 (I/M, 0.86); however, it was not statistically significant compared with that of the PBS group. Concentration appeared to play a more important role in efficacy of treatment in that $100 \mu \mathrm{mol} / \mathrm{L} \mathrm{R} 7 \mathrm{had}$ a greater effect on $\mathrm{I} / \mathrm{M}$ than $10 \mu \mathrm{mol} / \mathrm{L}$ of the $\mathrm{R} 9$ solution. Finally, the greatest effect on I/M was seen with the highest concentration and longest polymer length at $100 \mu \mathrm{mol} / \mathrm{L} \mathrm{R}$. At 0.53 , this group had an approximately $42 \%$ lower I/M ratio than the PBS or R5 control groups (Fig 6).

\section{Discussion}

Autologous vein remains one of the most useful conduits for revascularization of arterial occlusive disease. ${ }^{9}$ Unfortunately, the development of neointimal hyperplasia in these vein grafts is nearly universal and significantly limits their long-term patencies. In this study we found that the ex vivo treatment of vein graft segments with L-arginine polymers upregulates their production of NO and limits the development of neointimal hyperplasia in a length- and dose-dependent manner.

Previous reports have described various techniques of manipulating NO production by vascular tissues. These include gene transfer of endothelial $\mathrm{NOS}^{14}$ or 


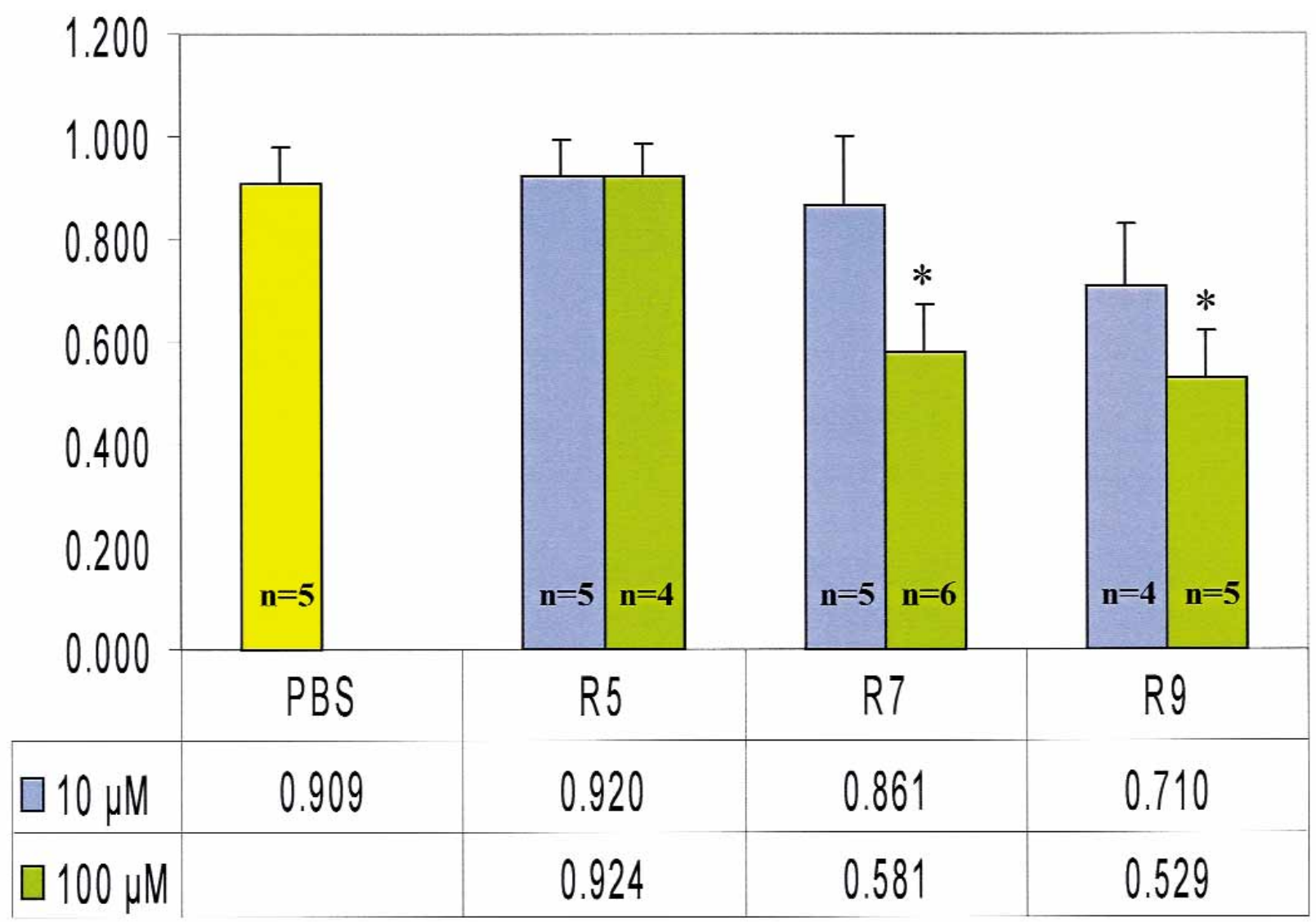

Fig 6. I/M ratio of treatment and control groups of 28-day-old vein interposition grafts. I/M is lowest in both 100 $\mu \mathrm{mol} / \mathrm{L}$ concentrations of R7 and R9. $* P<.001$ verus PBS, R5 $10 \mu \mathrm{mol} / \mathrm{L}$, and R5 $100 \mu \mathrm{mol} / \mathrm{L}$ (Bonferonni-corrected value).

iNOS, ${ }^{15}$ the regulation of intracellular cyclic adenosine monophosphate in combination with proinflammatory cytokines, ${ }^{16}$ and the dietary administration of the NOS substrate L-arginine. ${ }^{17}$

Dietary L-arginine reduces monocyte adhesion to vascular endothelium ${ }^{18}$ and is thought to mediate its effects by increasing production of NO by these infiltrating cells. ${ }^{19}$ The limitation of this mode of therapy, however, is the transient nature with which plasma Larginine levels appear to be manipulated by dietary supplementation. Jeremy, McCarron, and Sullivan ${ }^{19}$ found that despite continued oral administration of Larginine, plasma levels remained elevated only up to 14 weeks. This was apparently caused by modification of L-arginine homeostasis either through increased metabolism by arginase ${ }^{20}$ or enhanced urinary excretion..$^{21}$ The unsustainable elevations in plasma L-arginine content also correlated with a lack of a long-term benefit on vein graft endothelium-dependent vasodilatation in their model.
We have shown that single-dose therapy with L-arginine polymers of sufficient length and concentration inhibited the development of vein graft neointimal hyperplasia in an in vivo model. Sustained efficacy of other single-dose treatments has been noted previously. For example, ex vivo one-shot therapy with the NO donor S-nitroso- $N$-acetylpenicillamine successfully limited vein bypass neointimal formation in another study. ${ }^{8}$ It appears that certain treatments centered around the time of vessel perturbation with bypass grafting are able to influence cell migration, matrix production, and other variables associated with vascular remodeling, such that there are long-term inhibitory effects on neointima formation. ${ }^{22}$

Histologic examination of the human vein grafts treated with biotinylated L-arginine polymers demonstrated that there is considerable uptake deep into the vessel wall at appropriate lengths and concentrations. Similar ability for membrane translocation was also seen with the Tat protein of the HIV-1 virus, which is rich in argi- 
nine sequences (residues 49-57; RKKRRQRRR). ${ }^{23,24}$ Uemura and associates ${ }^{25}$ found that this process of polyarginine uptake appears to occur in vitro through an energy-dependent mechanism (incubation with azide abolishes uptake) and independent of the normal amino acid transporter mechanisms. Incubation with excess concentrations of L-arginine monomers failed to have any appreciable effect on polymer uptake. If the polymers did gain access to the cell through the same transport mechanisms as the amino acid monomer, one might expect competitive inhibition at these levels.

Once within the cell, arginine polymers can supply the substrate for iNOS. This inducible form of NOS is upregulated within smooth muscle cells of vessels subjected to either endothelial injury ${ }^{26}$ or proinflammatory cytokine stimulation. ${ }^{27}$ Measurement of nitrite in our stimulated vein segments did demonstrate a lengthdependent upregulation of the molecule that may be indicative of NO production by our treated venous grafts. These in vitro results were modest, however, and it is not known how long increases in NO would persist in treated arterialized vein segments.

The ultimate effect of L-arginine polymers on neointimal hyperplasia in vivo appears to be related to both the amount of substrate provided, as well as their ability to gain access to the cell. Neither concentration of R5 demonstrated any biologic effect on vein graft disease over PBS controls, and this is consistent with their lack of uptake. Interestingly, R7 at a concentration of $10 \mu \mathrm{mol} / \mathrm{L}$, while having fairly efficient intracellular access, did not have any appreciable effect on the development of increased $\mathrm{I} / \mathrm{M}$ ratio at 28 days. This suggests that although translocation is achieved, the ultimate effect on vein graft atherosclerosis also relies on the total substrate delivered to iNOS by the polymers. Indeed, the greatest effect on $\mathrm{I} / \mathrm{M}$ is achieved with the maximum dose of $100 \mu \mathrm{mol} / \mathrm{L}$ and the longest length of $\mathrm{R} 9$, respectively.

$\mathrm{NO}$ is an extremely diverse molecule, and focusing only on its effects on VSMC proliferation may be overly simplistic. The upregulation of iNOS under conditions of endothelial damage may represent a unique response to injury protective mechanism for vessels subjected to various stresses. For example, NO-mediated vasodilatation and inhibition of platelet aggregation may be an attempt by an injured vessel to stave off early vein graft thrombosis. ${ }^{28}$ Other investigators have demonstrated a protective role of exogenous NO delivery to hearts subjected to ischemia-reperfusion injury. ${ }^{29,30}$ Future directions of study will look at the effects of L-arginine polymer therapy in these important areas and further delineate the unique roles of $\mathrm{NO}$ in vascular graft biology.
Finally, arginine polymers may have intrinsic benefits independent of their effects on NO synthesis. As the photomicrographs depicting the biotinylated polypeptides suggest, R7 and R9 are capable of efficiently translocating deep into vessel walls. By linking the polymers to specific therapeutic agents, they may thus have a distinct utility as a carrier protein for directed treatments of various cardiovascular diseases.

\section{Conclusion}

In conclusion, L-arginine polymers of sufficient length and concentration can efficiently translocate across membranes of vascular cells in ex vivo treated vein grafts. By providing the substrate for iNOS production of NO, these constructs represent a novel therapy for the inhibition of neointimal hyperplasia, which may currently limit the long-term patency of venous bypass grafts.

Received for publication July 5, 2000; revisions requested Sept 12, 2000; revisions received Oct 5, 2000; accepted for publication Oct 26, 2000.

Address for reprints: Robert C. Robbins, MD, Falk Research Building, 2nd Floor, Stanford University Medical School, Stanford, CA 94305-5247 (E-mail: robbins@leland.stanford.edu).

\section{REFERENCES}

1. Canver CC. Conduit options in coronary artery bypass surgery. Chest 1995;108:1150-5.

2. Fitzgibbon GM, Kafka HP, Leach AJ, Keon WJ, Hooper GD, Burton JR. Coronary bypass graft fate and patient outcome: angiographic follow-up of 5,065 grafts related to survival and reoperation in 1,388 patients during 25 years. J Am Coll Cardiol 1996;28:616-26.

3. Motwani JG, Topol EJ. Aortocoronary saphenous vein graft disease: pathogenesis, predisposition, and prevention. Circulation 1998;97:916-31.

4. Sarkar R, Meinberg EG, Stanley JC, Gordon D, Webb RC. Nitric oxide reversibly inhibits the migration of cultured vascular smooth muscle cells. Circ Res 1996;78:225-30.

5. Best PJ, Hasdai D, Sangiorgi G, Schwartz RS, Holmes DR, Simari RD, et al. Apoptosis: basic concepts and implications in coronary artery disease. Arterioscler Thromb Vasc Biol 1999; 19:14-22.

6. Hecker M, Cattaruzza M, Wagner AH. Regulation of inducible nitric oxide synthase gene expression in vascular smooth muscle cells. Gen Pharmacol 1999;32:9-16.

7. Wu G, Morris SMJ. Arginine metabolism: nitric oxide and beyond. Biochem J 1998;336:1-17.

8. Fulton GJ, Davies MG, Barber L, Gray JL, Svendsen E, Hagen PO. Local effects of nitric oxide supplementation and suppression in the development of intimal hyperplasia in experimental vein grafts. Eur J Vasc Endovasc Surg 1998;15:279-89.

9. Okazaki J, Komori K, Kawasaki K, Eguchi D, Ishida M, Sugimachi K. L-Arginine inhibits smooth muscle cell proliferation of vein graft intimal thickness in hypercholesterolemic rabbits. Cardiovasc Res 1997;36:429-36. 
10. Davies MG, Dalen H, Kim JH, Barber L, Svendsen E, Hagen PO. Control of accelerated vein graft atheroma with the nitric oxide precursor: L-arginine. J Surg Res 1995;59:35-42.

11. Mitchell D, Kim DT, Steinman L, Fathman CG, Rothbard JB. Polyarginine enters cells more efficiently than other polycationic homopolymers. J Pept Res 2000;56:318-25.

12. Hill CM, Liu A, Marshall KW, Mayer J, Jorgensen B, Yuan B, et al. Exploration of requirements for peptide binding to HLA DRB1*0101 and DRB1*0401. J Immunol 1994;152:2890-8.

13. Cohen J, Evans TJ, Spink J. Cytokine regulation of inducible nitric oxide synthase in vascular smooth muscle cells. Prog Clin Biol Res 1998;397:169-77.

14. von der L, Gibbons GH, Morishita R, Lewis NP, Zhang L, Nakajima M, et al. Gene therapy inhibiting neointimal vascular lesion: in vivo transfer of endothelial cell nitric oxide synthase gene. Proc Natl Acad Sci U S A 1995;92:1137-41.

15. Shears LL, Kawaharada N, Tzeng E, Billiar TR, Watkins SC, Kovesdi I, et al. Inducible nitric oxide synthase suppresses the development of allograft arteriosclerosis. J Clin Invest 1997; 100:2035-42.

16. Koide M, Kawahara Y, Nakayama I, Tsuda T, Yokoyama M. Cyclic AMP-elevating agents induce an inducible type of nitric oxide synthase in cultured vascular smooth muscle cells: synergism with the induction elicited by inflammatory cytokines. J Biol Chem 1993;268:24959-66.

17. Cooke JP, Singer AH, Tsao P, Zera P, Rowan RA, Billingham ME. Antiatherogenic effects of L-arginine in the hypercholesterolemic rabbit. J Clin Invest 1992;90:1168-72.

18. Tsao PS, McEvoy LM, Drexler H, Butcher EC, Cooke JP. Enhanced endothelial adhesiveness in hypercholesterolemia is attenuated by L-arginine. Circulation 1994;89:2176-82.

19. Jeremy RW, McCarron H, Sullivan D. Effects of dietary L-arginine on atherosclerosis and endothelium-dependent vasodilatation in the hypercholesterolemic rabbit: response according to treatment duration, anatomic site, and sex. Circulation 1996;94: 498-506.

20. Castillo L, Ajami A, Branch S, Chapman TE, Yu YM, Burke JF, et al. Plasma arginine kinetics in adult man: response to an arginine-free diet. Metabolism 1994;43:114-22.

21. Southern LL, Baker DH. Performance and concentration of amino acids in plasma and urine of young pigs fed diets with excesses of either arginine or lysine. J Anim Sci 1982;55:857-66.

22. Morishita R, Gibbons GH, Horiuchi M, Ellison KE, Nakama $\mathrm{M}$, Zhang L, et al. A gene therapy strategy using a transcription factor decoy of the E2F binding site inhibits smooth muscle proliferation in vivo. Proc Natl Acad Sci U S A 1995;92:5855-9.

23. Vives E, Brodin P, Lebleu B. A truncated HIV-1 Tat protein basic domain rapidly translocates through the plasma membrane and accumulates in the cell nucleus. J Biol Chem 1997;272:16010-17.

24. Efthymiadis A, Briggs LJ, Jans DA. The HIV-1 Tat nuclear localization sequence confers novel nuclear import properties. J Biol Chem 1998;273:1623-8.

25. Uemura S, Fathman CG, Rothbard JB, Cooke, JP. Rapid and efficient vascular transport of arginine polymers inhibits myointimal hyperplasia. Circulation 2000;702:2629-35.

26. Hansson GK, Geng YJ, Holm J, Hardhammar P, Wennmalm A, Jennische E. Arterial smooth muscle cells express nitric oxide synthase in response to endothelial injury. J Exp Med 1994;180:733-8.

27. Chester AH, Borland JA, Buttery LD, Mitchell JA, Cunningham DA, Hafizi S, et al. Induction of nitric oxide synthase in human vascular smooth muscle: interactions between proinflammatory cytokines. Cardiovasc Res 1998;38:814-21.

28. Dusting GJ, Macdonald PS. Endogenous nitric oxide in cardiovascular disease and transplantation. Ann Med 1995;27:395-406.

29. Masini E, Salvemini D, Ndisang JF, Gai P, Berni L, Moncini M, et al. Cardioprotective activity of endogenous and exogenous nitric oxide on ischaemia reperfusion injury in isolated guinea pig hearts. Inflamm Res 1999;48:561-8.

30. Vinten-Johansen J, Sato H, Zhao ZQ. The role of nitric oxide and NO-donor agents in myocardial protection from surgical ischemic-reperfusion injury. Int J Cardiol 1995;50:273-81.

\section{Discussion}

Dr Robbin Cohen (Los Angeles, Calif). Saphenous vein grafts continue to be the most common conduits used by cardiac surgeons for coronary artery bypass grafting. They are usually readily available, can be removed endoscopically with minimal morbidity, and have excellent and immediate flow characteristics. Unfortunately, despite a tendency to become functionally arterialized, a term I learned from Craig Miller, they tend to respond to being interposed into the coronary arterial circulation with neointimal hyperplasia, usually followed by accelerated atherosclerosis and premature occlusion.

You and your colleagues in Dr Robbins' laboratory are to be commended for tackling the huge problem of vein graft failure to make the results of our most commonly used conduit more long lasting. Your ideal scenario goes something like this: vein grafts that are rendered ischemic and damaged by their excision react by producing iNOS. An easily administered single-dose bath of L-arginine in the appropriate size polymers at the time of excision readily crosses the cell membranes of vascular cells and serves as a substrate for NOS, and the result is the production of $\mathrm{NO}$, which fights off the proliferation of VSMCs, thereby inhibiting neointimal hyperplasia and presumably preventing the development of vein graft atherosclerosis. The study does provide potentially important data, which suggest that certain lengths of L-arginine polymers are taken up by vascular cells in a dose-dependent fashion. This is followed by an increase in NO production at 48 hours and a possible reduction in neointimal hypertrophy at 28 days, as measured with the I/M ratio. What the study does not do is justify your title. Although certainly a precursor, neointimal hyperplasia is not synonymous with atherosclerosis, nor was true atherosclerosis measured, observed, or really even mentioned in your article. Whereas your data warrant further investigation regarding the utility of bathing freshly harvested vein grafts in L-arginine, the assumption that it prevents atherosclerosis with the obvious benefits is premature. Still, your data are valuable, and I have 3 questions.

In your article there is about a $12 \%$ early occlusion rate of your vein grafts, which is not that dissimilar from what we see clinically. Did you look at those occluded grafts in a similar fashion to determine whether they produced increased $\mathrm{NO}$, and what was their neointimal hyperplasia situation like?

Dr Kown. Thank you for your comments, Dr Cohen. Those $12 \%$ that were determined to be occluded were deter- 
mined at the 28-day time point, well beyond the 48 hours that we used in our assays for NO production. It appears that these vessels were occluded because of early technical failure and not excessive neointimal hyperplasia in that histologic examination revealed only fibrous strands of scar tissue, with no evidence of any components of a vascular wall.

Dr Cohen. To perform your NO assay, you incubated vein sections in substances that are designed to upregulate iNOS production. Because your study makes ample L-arginine available to the vascular cell, to what extent is it actually dependent on that production of the iNOS? In other words, how reliable is that production? How much damage and ischemia has to occur for the production of NOS to occur, and how will that affect the production of NO down the line?

Dr Kown. There are 3 isoforms of NOS. One is the constitutive endothelial NOS, which is present in normal physiologic levels. If you did not have vessel perturbation associated with grafting, you would get a certain amount of NO produced by the vessel just by virtue of the fact that the endothelial NOS is present. This is easily discerned from how much production is created by a stressed vessel by adding a calcium ionophore because endogenous NOS is stimulated by calcium levels. When you do that, you see a slight incremental effect of NO produced by vein grafts; however, the predominant effect seen in our model when you have stressed vessels was from iNOS.

Dr Cohen. Finally, you chose as a single point in time, 28 days, to measure the I/M ratio and hence the degree of neointimal hyperplasia. Why not multiple time periods? Is this ratio on its way up or is it on its way down? Is this a long-lasting effect? Why did you not look at it a little bit more carefully?

Dr Kown. I think that is a good question. By studying our vein samples at 28 days for neointimal hyperplasia, we were trying to extrapolate how much actual vein graft disease occurs over the long term. The use of other models and time points would be a natural extension of these studies, but we believed that it was valuable to start with a snapshot of a single time point.

Dr Charles Brantigan (Denver, Colo). I would like to continue from where the previous discussant left off. Dr Cohen talked about providing arginine to eliminate the neointimal hyperplasia, and you talked about bathing the vein grafts in L-arginine or maybe flipping an on-and-off switch by presenting the bypass conduit with the L-arginine. How about a strategy that involves bathing the vein graft in arginine during the postoperative period by providing oral arginine to the animal or to the patient? There has been some evidence that suggests that giving L-arginine, which is available from a health food store, will in fact increase the levels of NO. We know that increasing the levels of NO decreases neointimal hyperplasia. Why not just provide something that is natural and free of side effects and thus provide some protection on a long-term basis? Have you investigated that strategy?

Dr Kown. We have seen from the literature that one way to upregulate $\mathrm{NO}$ is to provide dietary L-arginine. In one study with a hypercholesterolemic rabbit model, however, the authors found that for some reason, with oral administration of L-arginine, plasma levels were not maintained past 14 weeks. They attributed this to some sort of compensatory mechanism on arginine homeostasis by the body that led to increased urinary excretion and increased metabolism by arginase (which is the enzyme that will metabolize L-arginine). They thus found that they did not have a prolonged biologic effect past 14 weeks in that particular model.

\section{Authoritative}

The Journal of Thoracic and Cardiovascular Surgery is the most frequently cited thoracic/cardiovascular surgery journal in the Science Citation Index. An article in JTCVS is sited on average almost twice as often as those in the closest cardiothoracic journal. 